\title{
Aufbruchsstimmung im eIDAS-Ökosystem
}

Die Verordnung (EU) Nr. 910/2014 des Europäischen Parlaments und des Rates vom 23. Juli 2014 über elektronische Identifizierung (eID) und Vertrauensdienste, die gemeinhin als „elDAS-Verordnung" bekannt ist, eröffnet vielfältige Chancen für die vertrauenswürdige Abwicklung elektronischer Transaktionen - auch über Grenzen hinweg.

Wie im Beitrag "Notifizierung von elD-Systemen gemäß elDAS“ von Felix Bleckmann, Guido Frank, Michael Hoppe und Thomas Schnattinger näher erläutert, sind bereits zahlreiche Systeme zur elektronischen Identifizierung in Europa formal notifiziert, der Personalausweis mit Online-Ausweisfunktion wird bereits seit September 2018 Europaweit anerkannt und die grenzüberschreitende Anerkennung von vielen weiteren, bereits formal notifizierten, Identifizierungssysteme wird noch in diesem Jahr erfolgen. Neben der positiven Entwicklung der „notifizierten elD-Landschaft" gibt es weitere gute Neuigkeiten vom Personalausweis selbst, wie im Beitrag von Jens Bender, Björn Metzler und Niels Räth dokumentiert und darüber hinaus, wie von Houdeau et. al. bemerkt, weitere positive Impulse durch digitale Identitäten bei der Gestaltung des digitalen Wandels.

Aber nicht nur im Bereich der elektronischen Identifizierung gemäß der eIDAS-Verordnung gibt es zahlreiche vielversprechende Entwicklungen, sondern auch im Bereich der Vertrauensdienste und dort vor allem hinsichtlich der vergleichweise neuen Dienste für die Erstellung, Prüfung und langfristigen Bewahrung von elektronischen Signaturen und Siegeln. Wie im Beitrag "Neue APIs für das elDAS-Ökosystem“ von den bei OASIS DSS-X und ETSI ESI aktiven Experten (u.a. Ulrike Korte, Andreas Kuehne und Andrea Röck) beschrieben, entstehen hier Standards mit entsprechenden Webservice-Schnittstellen, die den Startpunkt für die Entwicklung einer umfassenderen „eIDAS-API“ bilden und die Entwicklung eines offenen elDAS-Ökosystems nachhaltig fördern können. Wie von Schwenk et. al. im Beitrag „Moderne Vertrauensdienste für vertrauenswürdige globale Transaktionen" näher erläutert, werden diese Standards aktuell im EU-Projekt „FutureTrust" umgesetzt und dort in unterschiedlichen Pilotanwendungen validiert, bevor am Ende des Projektes ausgewählte Komponenten als Open Source an die „eIDAS-Community“ übergeben werden sollen. Welchen Beitrag elektronische Signaturen, Siegel, Zeitstempel und Bewahrungsdienste generell und speziell in der Bundesverwaltung leisten können und wie eine kluges integriertes Gesamtsystem zur vertrauenswürdigen Digitalisierung der deutschen Verwaltung auf Basis der „eIDAS-API“ aussehen könnte, erläutert der Beitrag "Sichere Digitalisierung mit TR-RESISCAN und TR-ESOR“ von Ahmad et. al. Der Beitrag „Smarte Mobilität durch FiftyFifty Taxi“ der beiden Landräte Christian Meißner und Klaus Peter Söllner zeigt schließlich, dass mit der Kombination von elD- und Vertrauensdiensten neue Potenziale für die vertrauenswürdige Digitalisierung erschlossen werden können und dass die kluge Digitalisierung per Smartphone, Personalausweis und Co. keine Zukunftsmusik mehr ist, sondern selbst bei vergleichsweise kleinen Kommunen bereits gelebte Praxis!

Insgesamt scheinen die verschiedenen Beiträge des vorliegenden Hefts zu verdeutlichen, dass das große Potenzial der eIDAS-Verordnung nicht mehr nur auf dem Papier existiert, sondern dass es zunehmend auch in der Praxis genutzt wird. Um die aktuelle Aufbruchsstimmung im eIDAS-Ökosystem zu kultivieren und um die vielfältigen Impulse in eine positive Richtung zu lenken, haben sich führende europäische Verbände, Projekte und Expertenorganisationen aus dem Bereich eID und Vertrauensdienste in der gemeinnützigen „go.elDAS“-Initiative (https://go.eID.AS) zusammengeschlossen. Sie, liebe Leserinnen und Leser, sind herzlich eingeladen, sich dieser Initiative anzuschließen und die weitere Entwicklung des eIDAS-Ökosystems konstruktiv mitzugestalten - oder einfach die vielfältigen Chancen der elDAS-Verordnung als Anwender zu nutzen.

Tina und Detlef Hühnlein 